\title{
Evidence Based Diagnostic Approach to Tubal Factor Infertility
}

\author{
KHANUM S ${ }^{\mathrm{a}}$, AHMED JU ${ }^{\mathrm{b}}$, RAHIM MA ${ }^{\mathrm{c}}$, SULTANAN ${ }^{\mathrm{d}}$, BEGUM R ${ }^{\mathrm{e}}$
}

\begin{abstract}
Summary:
Infertility has been classified with respect to a number of parameters; prominent amongst which are time, causes, treatment cost and socio-cultural implications. The most widely accepted practical classification distinguishes between primary and secondary infertilities with a further sub classification into 3 clearly defined groups that include ovulatory dysfunction, fallopian tube compromise and male factor alongside an ambiguous and controversial group labeled as "unexplained infertility". In infertility work-up various procedures can be carried out in isolation or in different combination, depending on the indication. Other complimentary procedures can be done when specifically required. So, with infertility in general the diagnosis of tubal infertility should be tailored to the individual patient.
\end{abstract}

\section{Introduction:}

Infertility is defined as inability of a couple to conceive after one year of unprotected and adequate sexual intercourse. It is a public health problem affecting about $10-15 \%$ of couples. There are various causes responsible for infertility. Of them, tubo-peritoneal factors make up the most important single group of infertility. It accounts for $25-30 \%$ of female factor for infertility. ${ }^{1}$

a. Major (Dr.) Shakila Khanum, MBBS, MCPS, FCPS (Gynae), Classified Specialist, Department of Obstetrics \& Gynecology, Combined Military Hospital, Dhaka

b. Dr. Jamal Uddin Ahmed, MBBS, FCPS (Medicine), Assistant Professor, Internal Medicine, BIRDEM General Hospital

c. Dr. Muhammad Abdur Rahim, MBBS, FCPS (Medicine), Registrar, Internal Medicine, BIRDEM General Hospital

d. Dr. Nahid Sultana, MBBS, MS (Gynae), Assistant Professor, Department of Obstetrics \& Gynecology, BIRDEM General Hospital

e. Prof. Rahima Begum, MBBS, FCPS (Gynae), Head of the Department of Obstetrics \& Gynecology, BIRDEM General Hospital

Address of correspondence: Major (Dr.) Shakila Khanum, Classified Specialist, Department of Obstetrics \& Gynecology, Combined Military Hospital, Dhaka. E-mail: jmldollar@ gmail.com
Here is a review on an evidence based approach to diagnose the tubal factors for infertility.

Purpose of review:

The investigation for potential tubal disease is an essential step in the work-up of infertility. This review article will provide an overview of evidence based diagnosis of tubal factor infertility. The different diagnostic tools will be described together with discussion of different minimal access surgical approaches. We intend to highlight the capital and central role of minimal access procedures playing in the diagnosis of tubal infertility as well as to demonstrate the positive impact it has in the fight against infertility; thus redefining the classification and pathology.

(Birdem Med J 2014; 4(1): 33-37)

Tubal infertility includes the changes due to inflammation which involve the fallopian tube and its relation towards ovary in a way that will affect ovulation, the transport of egg, sperm or embryo or alter the function of the tube as the site of fertilization. Pelvic-peritoneal adhesions (mostly sequelae of previous infections) constitute the single most common class of tubal pathology responsible for tubal infertility. ${ }^{2}$ Other conditions include endometriosis, hydrosalpinx and proximal tube obstruction due to complications of endoluminal salpingitis. Bilateral tubal ligation carried out for definitive family planning can constitute a special category of tubal infertility in case of the need for reversal of tubal ligation. Rarely isolated external mechanical compression does give rise to infertility. Pelvic inflammation (PID) is the most common cause of tubal disease, representing more than $50 \%$ of the cases. ${ }^{2,3}$ It may affect the fallopian tube at multiple sites. After one episode of PID the rate of infertility has been estimated to be $11 \%$, which increases to $23 \%$ and $54 \%$ after two and three episodes respectively.

Tuboperitoneal disease results from both proximal and distal tubular diseases. 


\section{Proximal tubal disease}

In addition to PID, causes of proximal tubal disease include intratubal debris, congenital malformations, endometriosis and salpingitis isthmica nodosa (SIN). With SIN, diverticulae of the intramural or proximal isthmic endosalpinx enlarge and eventually obliterate the tubal lumen. ${ }^{4}$ SIN is bilateral in most affected patients and is associated with both infertility and ectopic pregnancy. ${ }^{5}$ Tubal polyps, found in approximately $11 \%$ of hysterectomy specimens, also can cause transient proximal tubal blockage. ${ }^{6}$ Similarly, endometriosis may affect the intramural portion of the tube, and is present at this site in $7-14 \%$ of patients with tubal factor infertility. $^{7}$

\section{Distal tubal disease}

Distal tubal disease is also caused by a multitude of factors, including salpingitis, antecedent elective sterilization, adhesions from previous surgery and endometriosis. Rock et al. classified distal tubal disease into mild, moderate or severe categories based on the size of hydrosalpinx, extent of adhesions, degree of fimbrial preservation and the appearance of the endosalpinx on hysterosalpingography (HSG). ${ }^{8}$ This classification has implications with respect to pregnancy and ectopic rates both before and after tubal reconstructive surgery.

\section{Recent findings:}

With recent advances in endoscopic procedures and other endoscopic assisted procedures, laparoscopic chromopertubation remains the gold standard in the diagnosis of tubal disease and hysterosalpingography is still widely used. Newer modality offer same advantages. Depending on the nature and degree of tubal dysfunction as well as the age and ovarian reserve of the patient, various treatments for tubal infertility are available. For proximal tubal obstruction, transcervical tubal cannulation with tubal flushing is a reasonable first approach. ${ }^{9}$ Surgical techniques for tubal repair, such as salpingostomy or fimbrioplasty for distal tubal obstruction, can provide good results. Still, tubal factor remains a major indication for in-vitro fertilization and embryo transfer, which bypasses the tubal problem altogether. In certain situations, such as the presence of hydrosalpinx, prophylactic surgery can be used in conjunction with in-vitro fertilization and embryo transfer.

\section{Diagnosis of Tubal Infertility: Minimal Access Procedures}

There are multiple approaches to the diagnosis of tubal infertility, including laparoscopy with chromopertubation, hysterosalpingography, sonohysterosalpingography, salpingoscopy, and chlamydial serology. Each diagnostic test has certain benefits and limitations; thus, the selection of a particular test or a combination of tests should be individualized to the patient.

\section{Hysterosalpingography (HSG):}

The diagnosis of tubal patency based on radiological findings is not considered a complete or an absolute diagnosis. X-ray gives an idea about the size and shape of the uterine cavity, the isthmus and the cervical canal when viewing anteroposterior or profile films. ${ }^{10} \mathrm{HSG}$ is widely used and has some advantages, including the lack of need for anesthesia, relative speed with which the procedure is completed, and a potential therapeutic effect with oil soluble contrast media. ${ }^{11}$ The therapeutic impact of HSG is, in part, due to the flushing of tubal debris. In addition, in-vitro studies have shown that oilbased flushing media prevents peritoneal mast cell phagocytosis of spermatozoa and increases fecundity in subfertile mice. A pathognomonic finding on HSG is seen with SIN, in which the contrast filled diverticular projections result in a radiographically honeycombed appearance. ${ }^{12}$ Hydrosalpinx also has a characteristic appearance; however, transvaginal ultrasound better evaluates the volume of the dilated tubes. ${ }^{13}$ HSG findings can be used to stage tubal disease and the appearance of the intraluminal mucosal architecture as a rugal pattern is a good prognostic factor for subsequent pregnancy. ${ }^{14,15}$ A potential limitation of HSG is tubal spasm, especially with elevated contrast injection pressure. Based on hysteroscopic tubal cannulation, it has been estimated that HSG may give a false positive diagnosis of proximal tubal obstruction $50 \%$ of the time. ${ }^{16}$ Lower pressure, the use of spasmolytic agents, such as glucagon, diazepam and terbutaline and follow-up imaging to assess contrast spillage following resolution of spasm have been proposed. ${ }^{3,17,18}$. However, intermittent tubal obstruction during HSG may suggest underlying tubal pathology, especially in the setting of low injection pressures, and thus the value of spasmolytic agents may be limited. ${ }^{3}$ Furthermore, the efficacy of these 
agents with respect to reversal of tubal spasm remains to be established. ${ }^{19,20}$ A recent study suggested that unilateral cornual obstruction may be resolved in more than $50 \%$ of patients by rotating the patient so that the obstructed tube is in a more inferior position. ${ }^{21}$

\section{Endoscopic evaluation:}

Developments within the past two decades in the domain of optics, video-imaging and endoscopy have completely changed the diagnostic approach for tubal infertility. The tube can now be examined directly in real time under magnification and in its natural habitat under physiological conditions contrary to HSG. ${ }^{22}$ Exploration of the tube is now more extensive with more dynamic investigative maneuvers and with less risks, incidents and accidents.

Magnification in endoscopic diagnosis has significantly reduced inter-observer variation in describing lesions unlike in clinical and biological investigations and $\mathrm{HSG}^{23}$ All of the above characteristics and inherent advantages has positioned endoscopy as the gold standard in the diagnostic management of tubal infertility. ${ }^{24}$ Catalano GF et al using salpingoscopy showed that the state of the tubal mucosa was the most important prognostic factor for fertility with rates ranging between 60 and $70 \%{ }^{25}$

\section{a). Laparoscopy}

Laparoscopy provides useful information on the external aspect of the uterus, its size, shape and the appearance of the serosa suggesting the presence of a former inflammatory process. The cornua should be inspected very carefully, looking for signs of rigidity, abnormal surface vessels, thickening of the cornua or isthmic part of the tube due either to endometriosis or post infectious changes. The extreme pathology is described as salpingitis isthmica nodosa. Adhesions should be looked at as described traditionally. Laparoscopy with chromopertubation has been the gold standard for investigating tubal patency. ${ }^{26} \mathrm{~A}$ meta-analysis comparing the accuracy of HSG to diagnostic laparascopy with chromopertubation in the diagnosis of tubal pathology found point estimates of $65 \%$ and $83 \%$ for sensitivity and specificity respectively. ${ }^{26}$ This analysis was limited by the inclusion of only retrospective studies in which patients underwent both HSG and diagnostic laparoscopy, thus excluding those patients who conceived after HSG. ${ }^{26}$ However, in another study in which laparoscopy was performed on all subjects on the day after HSG, the latter had a similar sensitivity and specificity of $54 \%$ and $83 \%$, respectively. 27 The inability of HSG to detect peritubal adhesions limits its sensitivity as a diagnostic test. ${ }^{26}$ Still, laparoscopic chromopertubation is not a perfect gold standard as presumed tubal obstruction may be due to differences in resistance between the tubes, spasm or technical failure. ${ }^{26}$

\section{b). Salpingoscopy}

Salpingoscopy is an endoscopic approach for the diagnosis of intraluminal tubal damage. During laparoscopy, a rigid salpingoscope is inserted into the distal tubes, allowing for visualization of the ampullary tubal mucosa. Although the degree of tubal mucosal damage, defined as the extent of adhesions between mucosal folds or the flattening of mucosal folds, does not necessarily correlate with that of periadnexal adhesions, it does directly correlate with poor pregnancy outcome. ${ }^{28-30}$ Thus, while laparascopic adhesiolysis can restore external tubal anatomy to facilitate oocyte pickup, tubal function may still be compromised if the endosalpinx is damaged. ${ }^{31}$ This was clearly demonstrated in a study of 51 patients with periadnexal adhesions or hydrosalpinx, who underwent adhesiolysis or salpingostomy, respectively, in addition to salpingoscopy. ${ }^{28}$ Patients with normal tubal mucosa at the time of laparoscopic surgery had $71 \%$ and $64 \%$ cumulative pregnancy rates in the adhesiolysis and salpingostomy groups, respectively, while those with intraluminal tubal damage had no intrauterine pregnancies. $^{28}$

\section{Sonographic evaluation:}

Sonohysterosalpingography with the use of contrast medium is an alternative to HSG for assessing tubal patency. ${ }^{32}$ Although the sonographic images are inferior to those obtained fluoroscopically, abdominal ultrasound has a sensitivity and specificity of $100 \%$ and $96 \%$ respectively, while transvaginal ultrasound is $89 \%$ sensitive, yet $100 \%$ specific in the detection of tubal blockage. ${ }^{23,33} \mathrm{~A}$ meta-analysis of the latter, which included three studies consisting of over 1000 patients, concluded that this method is superior to HSG and comparable to laparascopic chromopertubation. ${ }^{24}$ In aircontrast sonohysterography (SHG), a small amount of air is injected into the uterine cavity, and the passage of 
bubbles through the tubes is assessed. ${ }^{25}$ Jeanty et al. found that air-contrast SHG was in agreement with laparoscopic chromopertubation $79.4 \%$ of the time. ${ }^{25} \mathrm{~A}$ sensitivity and specificity of $85 \%$ and $87 \%$ were calculated. The main advantage of sonohysterographic methods is their ability to detect uterine abnormalities, such as fibroids and polyps, which may also impair fertility. While air contrast SHG was able to detect 85\% of anomalies, HSG detected only $6 \% .{ }^{34}$. Furthermore, air-contrast SHG is inexpensive, quick and better tolerated by patients than HSG. ${ }^{35}$

\section{Serological evaluation:}

Chlamydia antibody testing (CAT) to assess previous infection with Chlamydia trachomatis is a cost effective and non-invasive approach to evaluate fallopian tubes. ${ }^{36}$ There are four different serological techniques for C. trachomatis assessment: Microimmunofluorescence (MIF), immunofluorescence (IFT), immunoperoxidase assay, and enzyme-linked immunosorbent assay (ELISA). MIF detects typespecific immunoglobulin $\mathrm{G}$ antibodies, while the other methods detect antibodies against a much broader antigen found after both $C$. trachomatis and $C$. pneumonia exposure; thus, sensitivity is increased at the expense of specificity. ${ }^{36} \mathrm{Mol}$ et al. performed a metaanalysis comparing CAT with HSG for the diagnosis of tubal occlusion, using laparoscopic chromopertubation as the gold standard. ${ }^{36}$ They found that MIF had a sensitivity and specificity of 75\%, while the other tests were less specific. As the discriminative ability of CAT was comparable to that of HSG, the authors concluded that CAT can be used instead of HSG in the initial screening for tubal disease. ${ }^{36}$ Yet, CAT is limited by its inability to provide anatomical information with respect to the uterus or tubes and its lack of a potentially therapeutic effect. ${ }^{36}$ Antibody testing against chlamydia heat shock protein 60 (chsp60), a marker of chronic inflammation, has also been proposed as there is evidence that the antibody response to chsp60 predicts subsequent risks of tubal infertility and ectopic pregnancy ${ }^{37-39}$. In a prospective case control study using either HSG or laparoscopic chromopertubation for comparison, Claman et al. found $44 \%$ sensitivity and 92\% specificity for chsp60 antibody, while sensitivity and specificity of CAT with MIF were 63\% and 54\% respectively. ${ }^{37}$ In another study, using only laparoscopic chromopertubation as a reference, chsp60 had a lower sensitivity and specificity (51\% and 85\%) than CAT (54\% and 92\%). ${ }^{40}$ When both chsp60 testing and CAT were combined, the positive predictive value with respect to the detection of true tubal disease was increased. ${ }^{40}$

\section{Conclusion:}

Among various diagnostic procedures for tubal factor infertility, laparoscopic chromopertubation remains the gold standard. But other diagnostic modalities, including HSG, SHG, salpingoscopy, and chlamydia serology can assess the tubal patency and may offer some advantages in particular situations. The selection of investigation procedure should be individualized depending on age, duration of infertility, pretest probability of the cause and availability of investigation facility especially in resource poor setting.

\section{References:}

1. Serafini P, Batzofin J. Diagnosis of female infertility. J Reprod Med 1989; 34:29-40.

2. Grant A. Infertility surgery of the oviduct. Fertil Steril 1971; 22:496-503.

3. Honore GM, Holden AE, Schenken RS. Pathophysiology and management of proximal tubal blockage. Fertil Steril 1999; 71:785-95.

4. McComb PF, Rowe TC. Salpingitis isthmica nodosa: evidence it is aprogressive disease. Fertil Steril 1989; 51:542-45.

5. Creasy J, Clark RL, Cuttino JT, Groff T. Salpingitis isthmica nodosa: radiologic and clinical correlates. Radiology 1985; 154:587-600.

6. Lisa J, Gioia J, Rubin I. Observations of the interstitial portion of the fallopian tube. Surg Gynecol Obstet 1954; 99:159-69.

7. Fortier J, Haney A. The pathologic spectrum of uterotubal junction obstruction. Obstet Gynecol 1985; 65:93-98.

8. Rock JA, Katayama P, Martin EJ, et al. Factors influencing the success of salpingostomy techniques for distal fimbrial obstruction. Obstet Gynecol 1978; 52:591-96.

9. Kumpe DA, Zwerdlinger SC, Rothbarth LJ, et al. Proximal fallopian tube occlusion: diagnosis and treatment with transcervical fallopian tube catheterization. Radiology 1990; 177:183-87.

10. Shah SM, Towobola OA, Masihleho M. Diagnosis of fallopian tube patency. East Afr Med J. 2005 Sep;82(9):457-62.

11. Watson A, Vandekerckhove P, Lilford R, et al. A metaanalysis of the therapeutic role of oil soluble contrast media at hysterosalpingography: a surprising result? Fertil Steril 1994; 61:470-77. 
12. Jenkins C, Williams S, Schmidt G. Salpingitis isthimic nodosa: a review of the literature, discussion of clinical significance, and consideration of patient management. Fertil Steril 1993; 60:599-607.

13. De Witt W, Gowrising CJ, Kuik DJ, et al. Only hydrosalpinges visible on ultrasound are associated with reduced implantation and pregnancy rates after IVF. Hum Reprod 1997; 12:170.

14. Donnez J, Casanas-Roux F. Prognostic factors of fimbrial microsurgery. Fertil Steril 1986; 46:1089-92.

15. Young PE, Egan JE, Barlow J, Mulligan WE. Reconstructive surgery for infertility at the Boston Hospital for Women. Am J Obstet Gynecol 1970; 108:1092-97.

16. Novy M, Thurmond AS, Patton P, Uchida BT, Rosch J. Diagnosis of cornual obstruction by transcervical fallopian tube cannulation. Fertil Steril 1988; 50:434-40.

17. Thurmond AS, Novy M, Rosch J. Terbutaline in diagnosis of interstitial fallopian tube obstruction. Invest Radiol 1988; 23:209-10.

18. William D. A new hysterographic approach to the evaluation of tubal spasm and spasmolytic agents. Fertil Steril 1983; 39:105-7.

19. Winfield AC, Pittaway D, Maxson W, et al. Apparent cornual occlusion in hysterosalpingography: reversal by glucagon. Am J Roentgenol 1982; 139:525-27.

20. Cooper JM, Rigberg HS, Houck R, Aiken M. Incidence, significance, and remission of tubal spasm during attempted hysteroscopic tubal sterilization. J Reprod Med 1985; 30:39-42.

21. Hurd WW, Wyckoff ET, Reynolds DB, et al. Patient rotation and resolution of unilateral cornual obstruction during hysterosalpingography. Obstet Gynecol 2003; 101:1275-78.

22. Hamilton JA, Larson AJ, Lower AM. Evaluation of the performance of hysterosalpingo-contrast sonography in 500 consecutive, unselected, infertile women. Hum Reprod 1998; 13:1519-26.

23. Schlief R, Diechert U. Hysterosalpingo-contrast sonography of the uterus and fallopian tubes: results of a clinical trial of a new contrast medium in 120 patients. Radiology 1991; 178:213-15.

24. Holz K, Becker R, Schurmann R. Ultrasound in the investigation of tubal patency. A meta-analysis of three comparative studies of Echovist-200 including 1007 women. Zentralbl Gynakol 1997; 119:366-73.

25. Jeanty P, Besnard S, Arnold A, et al. Air-contrast sonohysterography as a first step assessment of tubal patency. J Ultrasound Med 2000; 19:519-27.

26. Swart P, Mol B, van der Veen F, et al. The accuracy of hysterosalpingography in the diagnosis of tubal pathology: a meta-analysis. Fertil Steril 1995; 64:486-91.
27. Swolin K, Rozencrantz M. Laparoscopy versus hysterosalpingography in sterility investigations, a comparative study. Fertil Steril 1972; 23:270-73.

28. Marana R, Catalano GF, Muzii L, et al. The prognostic role of salpingoscopy in laparoscopic tubal surgery. Hum Reprod 1999; 14:2991-95.

29. Marchino GL, Gigante V, Gennarelli G, et al. Salpingoscopic and laparoscopic investigations in relation to fertility outcome. J Am Assoc Gynecol Laparosc 2001; 8:21821.

30. De Bruyne F, Puttemans P, Boeck W, Brosens I. The clinical value of salpingoscopy in tubal infertility. Fertil Steril 1989; 51:339-40.

31. Marana R, Catalano GF, Muzii L. Salpingoscopy. Curr Opin Obstet Gynecol 2003; 15:333-36.

32. Fleisher AC, Vasquez JM, Cullinam JA. Sonohysterography combined with sonosalpingography: Correlation with endoscopic findings in infertility patients. J Ultrasound Med 1997; 16:381-85.

33. Richman TS, Viscomi GN, Decherney AH, et al. Fallopian tube patency assessed by ultrasound following fluid injection. Radiology1984; 152:507-10.

34. Chenia F, Hofmeyr GJ, Moolla S, et al. Sonographic hydrotubation using agitated saline: A new technique for improving fallopian tube visualization. Br J Radiol 1997; 70:833-36.

35. Exacoustos C, Zupi E, Carusotti C, et al. Hysterosalpingocontrast sonography compared with hysterosalpingography and laparoscopic dye pertubation to evaluate tubal patency. J Am Assoc Gynecol Laparosc 2003; 10:367-72.

36. Mol BWJ, Dijkman B, Wertheim P, et al. The accuracy of serum chlamydial antibodies in the diagnosis of tubal pathology: a meta-analysis. Fertil Steril 1997; 67:103137.

37. Claman P, Honey L, Peeling R, et al. The presence of serum antibody to the chlamydial heat shock protein (chsp60) as a diagnostic test for tubal factor infertility. Fertil Steril 1997; 67:501-4.

38. Toye B, Laferrier C, Claman P, et al. Association between antibody to the chlamydial heat-shock protein and tubal infertility. J Infect Dis 1993; 168:1236-40.

39. Brunham RC, Peeling R, MacLean I, et al. Chlamydia trachomatis-associated ectopic pregnancy: serologic and histologic correlates. J Infect Dis 1992; 165:1076-1081.

40. Den Hartog J, Land J, Stassen F, et al. Predictive value of chsp60 antibodies in screening for tubal subfertility. Abstracts of the 19th Annual Meeting of ESHRE, Madrid 2003; 18(1): 83. 